\title{
Therapeutic approaches for lysosomal storage diseases
}

\author{
Gregory M. Pastores
}

Abstract: The lysosomal storage disorders (LSDs) comprise a heterogeneous group of inborn errors of metabolism characterized by tissue substrate deposits, most often caused by a deficiency of the enzyme normally responsible for catabolism of various byproducts of cellular turnover. Individual entities are typified by involvement of multiple body organs, in a pattern reflecting the sites of substrate storage. It is increasingly recognized that one or more processes, such as aberrant inflammation, dysregulation of apoptosis and/or defects of autophagy, may mediate organ dysfunction or failure. Several therapeutic options for various LSDs have been introduced, including hematopoietic stem cell transplantation, enzyme replacement therapy and substrate reduction therapy. Additional strategies are being explored, including the use of pharmacological chaperones and gene therapy. Most LSDs include a variant characterized by primary central nervous system (CNS) involvement. At present, therapy of the CNS manifestations remains a major challenge because of the inability to deliver therapeutic agents across the intact blood-brain barrier. With improved understanding of underlying disease mechanisms, additional therapeutic options may be developed, complemented by various strategies to deliver the therapeutic agent(s) to recalcitrant sites of pathology such as brain, bones and lungs.

Keywords: enzyme replacement therapy, gene therapy, lysosomal storage disease, pharmacologic chaperone, substrate reduction therapy

\section{Introduction}

Knowledge of lysosomal storage disorders (LSDs) has evolved, from recognition of the clinical manifestations suggestive of a storage disease to characterization of the biochemical and subsequently molecular basis. In parallel, the management of affected individuals has advanced, from symptomatic or palliative care to disease-specific treatments that lead to substantial clearance of tissue deposits and amelioration of disease. Several recent reviews provide descriptions of the phenotype associated with individual LSDs and the various methods of diagnostic confirmation [Jardim et al. 2010; Hopwood and Ebner, 2009]. In the current review we focus on aspects relating to therapeutic strategies.

The pathologic hallmark of LSDs is the presence of membrane-enclosed tissue deposits, derived from the accumulation within the lysosome of several byproducts of cellular turnover that are not completely metabolized. The most common cause is a deficiency of the enzyme normally responsible for substrate catabolism; although in some cases, lysosomal storage may develop because of a deficiency of a cofactor required for substrate hydrolysis, or defective trans-membrane protein involved with facilitating substrate transport out of the lysosome [ParkinsonLawrence et al. 2010].

Deficient enzyme activity may arise as a consequence of mutations in the cognate gene, which results in either the complete obliteration of or significant reduction in protein activity. In the first instance, affected individuals tend to have earlier onset of symptoms and a disease characterized by a rapidly progressive course, often with primary central nervous system (CNS) involvement. In patients who exhibit residual enzyme activity, clinical manifestations may not be apparent until later in life. The latter situation partly explains the delay in diagnosis that is often seen in clinical practice, particularly among those without a positive family history and in cases wherein disease expression may be atypical.
Ther Adv Endocrinol Metab

(2010) 1(4) 177-188 DOI: $10.1177 /$ 2042018810384429

(C) The Author(s), 2010 Reprints and permissions: http://www.sagepub.co.uk/ journalsPermissions.nav

Correspondence to: Gregory M. Pastores, MD Department of Neurology and Pediatrics, NYU School of Medicine, 403 East 34th Street, New York, NY 10016, USA gregory.pastores nyumc.org 
Several therapeutic strategies have been introduced or proposed for distinct LSDs (Table 1). Essentially, the primary aim of treatment is the preservation or restoration of organ function, which can be achieved by preventing or reducing tissue substrate build up. Clinically proven approaches include the restoration of intracellular activity through crosscorrection, by transplanting healthy donor cells or infusion of the relevant exogenous enzyme, and by substrate synthesis inhibition. Investigational options include the use of pharmacologic chaperones and gene therapy. In this regard, animal models of disease have been invaluable in establishing a preclinical proof of concept [Haskins, 2007].

\section{Cellular transplantation}

Earlier, it had been shown that coculture of affected and healthy skin fibroblasts resulted in the clearance of lysosomal storage in the enzyme-deficient cells [Fratantoni et al. 1968]. The latter phenomenon of metabolic crosscorrection was subsequently attributed to the release of the relevant enzyme from healthy cells and its uptake by affected cells; this observation eventually led to recognition of the pathways involved with internalization of the enzyme. Ultimately, these studies provided the rationale for bone marrow transplantation, and subsequently the use of cord blood stem cell transplantation (HSCT) for several LSDs. To achieve maximal enzyme activity following HSCT, donor cells are preferably obtained from healthy noncarrier individuals.

Allogeneic HSCT has been undertaken in patients with various types of LSDs, but currently performed primarily for those with Krabbe disease (globoid cell leukodystrophy) and mucopolysaccharidosis type I (MPS IH; Hurler disease) [Prasad and Kurtzberg 2010a; Malm et al. 2008; Orchard et al. 2007]. Krabbe $(\beta$-galactocerebrosidase deficiency) and Hurler ( $\alpha$-L-iduronidase deficiency) disease are LSDs associated with primary CNS involvement. For these conditions, the benefits of HSCT result from donor-derived macrophages and microglia that populate the brain and lead to correction of the enzyme deficiency within neurons and glia [Krivit et al. 1995]. Beneficial effects may also accrue from changes in the cellular microenvironment leading to immune modulation and the mitigation of inflammation [Wada et al. 2000]. HSCT may also mediate nonhematopoietic cell regeneration or repair. HSCT has also been undertaken in patients with Hunter (iduronate-2-sulfatase) and Sanfilippo syndrome without clear-cut benefit; the basis for these

Table 1. Therapeutic approaches for lysosomal storage disorders (LSDs).

\begin{tabular}{|c|c|}
\hline Therapeutic option & Candidate diseases \\
\hline $\begin{array}{l}\text { 1. Bone marrow or umbilical cord blood } \\
\text { transplantation }\end{array}$ & $\begin{array}{l}\text { - Hurler (MPS IH), Maroteaux-Lamy (MPS VI), globoid-cell } \\
\text { leukodystrophy (Krabbe disease), metachromatic leuko- } \\
\text { dystrophy (MLD), } \alpha \text {-mannosidosis }\end{array}$ \\
\hline 2. Enzyme replacement therapy & $\begin{array}{l}\text { - Gaucher disease (GD), Fabry disease (FD), Hurler-Scheie } \\
\text { (MPS I), Hunter (MPS II) syndrome, Maroteaux-Lamy } \\
\text { (MPS VI), Pompe disease (GSD II). Investigational: recom- } \\
\text { binant acid and sphingomyelinase (Niemann-Pick B) }\end{array}$ \\
\hline 3. Substrate reduction therapy & $\begin{array}{l}\text { - Approved for GD, Niemann-Pick type C (miglustat); } \\
\text { Investigational for FD, late-onset GM2-gangliosidosis } \\
\text { (LOTS), Sandhoff disease. Investigational drugs: eliglu- } \\
\text { stat (GD) and genistein (MPS) }\end{array}$ \\
\hline 4. Substrate depletion therapy & - Cystinosis \\
\hline $\begin{array}{l}\text { 5. Substrate modulation (optimization) } \\
\text { therapy* }\end{array}$ & - MPS disorders \\
\hline $\begin{array}{l}\text { 6. Chaperone-mediated enzyme } \\
\text { enhancement* }\end{array}$ & - GD, FD and GSD II \\
\hline 7. Premature stop codon read through* & - MPS I \\
\hline 8. Gene therapy* & $\begin{array}{l}\text { - Almost all LSDs, mainly mouse models, except for GD } \\
\text { wherein early human trials were done }\end{array}$ \\
\hline 9. Antisense splicing modulation therapy* & - Niemann-Pick C \\
\hline
\end{tabular}


observations is unclear [Lau et al. 2010; Araya et al. 2009].

Procedural morbidity and mortality risks associated with HSCT have limited its general application. Engraftment and survival rates range from $63 \%$ to $90 \%$. Also, outcome among survivors may be partial, and influenced by pre-existing pathology or disease stage at the time of the transplant. For instance, after HSCT a significant proportion of children with MPS IH still exhibit short stature and about a third have thyroid disease [Prasad and Kurtzberg, 2008]. It is hoped that the introduction of newborn screening for treatable LSD may facilitate the introduction of early intervention, although it may be that there are intrinsic limitations to HSCT attributable to disease processes that are not sufficiently addressed by metabolic crosscorrection and/or immune modulation.

Advances in HSCT, particularly those aimed at reducing graft versus host disease, may lead to a reduction in procedure-related risks [Prasad and Kurtzberg, 2010b]. In addition, the potential to manipulate donor cells to enable overexpression of the cognate enzyme or increase the number of donor cells is being explored [Miyake et al. 2010; Wang et al. 2009].

\section{Enzyme replacement therapy}

Enzyme replacement therapy (ERT) has proved to be the most successful approach to treat patients with LSDs, particularly in the case of
Gaucher disease (GD), although ERT has not been able to prevent or reverse complications related to primary CNS involvement in the neuropathic subtypes (i.e. types 2 and 3 GD) [Schiffmann et al. 1997].

Intravenous ERT has been given to patients with GD, Fabry disease, Pompe disease and MPS I, II and VI with resultant improvements (Table 2), although, in some cases, one encounters amelioration or stabilization rather than reversal of the disease process [van der Ploeg et al. 2010; Mehta et al. 2009]. For instance, patients with Fabry disease who have severely impaired renal function and cardiac hypertrophy experience further deterioration on ERT, although there may be modification of the temporal course [Caballero et al. 2010]. In the MPS disorders, growth continues to be significantly stunted in most patients, even when therapy is started prior to puberty. These observations may reflect delay in diagnosis and the institution of therapy when disease stage has advanced. It is also possible that involvement of multiple cells types, including those with limited regenerative capacity, may represent an extra hurdle. In addition, there may be mechanisms of disease that may not be fully reversible or controlled by efforts such as ERT, which is directed solely at substrate clearance [Bellettato and Scarpa, 2010].

ERT has also been used in MPS I patients prior to and during HSCT, and it appears to reduce

Table 2. Enzyme replacement therapy.

\begin{tabular}{|c|c|c|}
\hline Disease & Enzyme & Therapeutic responses \\
\hline 1. Gaucher & - $\beta$-glucosidase & $\begin{array}{l}\text { - Improved blood counts, reduction of liver and spleen } \\
\text { volume, improved bone density at the lumbar spine }\end{array}$ \\
\hline 2. Fabry & - $\alpha$-galactosidase A & $\begin{array}{l}\text { - Partially mitigates neuropathic pain and gastrointes- } \\
\text { tinal discomfort, modifies course of renal disease in } \\
\text { mild to moderately CKD, reduces left ventricular } \\
\text { hypertrophy; does not appear to significantly alter } \\
\text { incidence of stroke }\end{array}$ \\
\hline 3. MPS disorders & & $\begin{array}{l}\text { - Reduced urinary GAG excretion; reduced liver and } \\
\text { spleen volume, improvement in FVC, increased dis- } \\
\text { tance travelled in time walk }\end{array}$ \\
\hline - Type I & - $\alpha$-L-iduronidase & \\
\hline - Type II & - Iduronate-2-sulfatase & \\
\hline - Type VI & - Arylsufatase B & \\
\hline 4. Pompe & - $\alpha$-glucosidase & $\begin{array}{l}\text { - Resolution of cardiomyopathy (infantile-onset cases); } \\
\text { stabilization or improvement in muscle strength; } \\
\text { respiratory improvement }\end{array}$ \\
\hline
\end{tabular}


respiratory complications after transplantation [Tolar et al. 2008].

ERT is generally well tolerated, although some patients have experienced fever, rigors, chills and urticaria. These adverse events which may develop during the infusion can be readily mitigated by slowing the rate of infusion and the administration of premedications, such as antihistamines and steroids. However, anaphylactic episodes have been described, and close monitoring of patients on ERT is thus warranted, particularly prior to transition to home infusion.

Antibodies have formed in a proportion of ERT-treated patients, although there is uncertainty regarding their influence on therapeutic outcome [Starzyk et al. 2007; Linthorst et al. 2004]. It appears a majority of the antibodies formed against the recombinant enzymes are nonneutralizing, although a fraction of antibodies that have been detected either neutralize enzyme activity or inhibit its tissue uptake. The latter situation is dramatically evident in infantile cases of Pompe disease, wherein survival among treated patients with null mutations is not significantly effected by ERT [Kishnani et al. 2010]. It has been suggested immunomodulation may limit the adverse effects of antibody formation, although the long-term safety of this approach and resultant benefit remain to be established [Sun et al. 2007].

In practical terms, ERT requires a commitment to regular intravenous infusion of the recombinant product and manufacturing costs are likely significantly higher when compared with the production of small molecule chemical drugs. Moreover, the benefits derived from intravenous ERT have primarily been confined to systemic (i.e. extraneurologic) organs. This observation is likely because only small amounts of intravenous proteins can cross the blood-brain barrier (BBB) by transcytosis. Thus, investigations are actively pursuing various strategies for directly delivering the enzyme into the cerebrospinal fluid or brain parenchyma, or modifying the recombinant protein so that it can be readily taken across the BBB [Hemsley et al. 2009]. Direct injection into the CNS of the recombinant enzyme has been attempted in animal models of MPS I and IIIA and has led to improvements [Dickson et al. 2007; Savas et al. 2004]. The potential safety and efficacy of this approach in human patients is being actively explored
[Munoz-Rojas et al. 2008]. Attempts to modify the cognate protein to enhance its uptake across the BBB have included the fusion of the gene for glucocerebrosidase (enzyme deficient in GD) with apolipoprotein $\mathrm{B}(\mathrm{ApoB})$, and intravascular delivery with a lentiviral vector. The resultant protein product was secreted from transduced cells and shown to be taken up via ApoB receptors on the BBB [Spencer and Verma, 2007]. In separate experiments involving the mouse model of MPS VII, inactivation of the exposed carbohydrate recognition markers by sodium metaperiodate/sodium borohydride led to effective deglycosylation of $\beta$-glucoronidase and greater brain uptake compared with unmodified enzyme; as a consequence there was marked reduction of neuronal storage [Grubb et al. 2008].

\section{Small-molecule-based therapies}

Several small molecules have been identified which exhibit properties that make them suitable as prospective treatment options for LSDs; these advantages include an oral route of administration, wide volume of distribution and access to potentially sequestered sites (e.g. across the BBB) and the absence of antibody formation to the drug (which in some cases reduces the benefit derived from enzyme replacement) and the need for immunomodulation (e.g. in the case of HSCT). Obviously, the safety and efficacy of these drugs will need to be closely monitored in light of the chronic exposure that will be necessary to maintain disease control. In particular, drugs that are internally processed may compete with the metabolism of other medications the patient may be on, or exhibit interactions with other agents or physiologic processes that can be potentially deleterious. In addition, nonspecificity of drug action may involve off-target effects and raise additional concern about its safety. With certain investigational approaches, a strategy will need to be developed to screen patients with particular mutations that may be corrected by a premature stop codon read-through option or whose misfolded protein product may be rescued by pharmacologic chaperones (explained in the following). Ultimately, in vitro screening strategies to identify potentially responsive patients must be correlated with in vivo effects that are predictive of positive clinical outcomes.

\section{Substrate reduction therapy}

Substrate reduction therapy (SRT) is designed to partially reduce the synthesis of the substrate or 
its precursor, to approximate the residual degradable capacity of the mutant enzyme and thereby strike a metabolic balance within the cell [Platt and Jeyakumar, 2008]. This approach was initially demonstrated to result in clinical benefit in patients with type 1 GD given miglustat, an iminosugar which inhibits the activity of glucosyceramide synthase [Pastores et al. 2005; Cox et al. 2000]. Miglustat's mechanism of action, by limiting precursor synthesis, reduces not only glucocerebroside accumulation but also the buildup of GM2 ganglioside (i.e. the offending lipid in Tay-Sachs disease [TSD], Sandhoff disease and Niemann-Pick type C [NPC]). In patients with NPC, improved supranuclear gaze palsy and swallowing has been observed following miglustat treatment [Patterson et al. 2007]. Unfortunately, the use of miglustat in patients with TSD has not been shown to significantly alter disease course in infantile and late onset cases [Maegawa et al. 2009; Shapiro et al. 2009]. In patients with Sanhoff disease, there are indications of potential benefit, although this is a matter which requires further investigation [Tallaksen and Berg, 2009; Wortmann et al. 2009].

Miglustat has been used as a maintenance therapy in selected patients, and a significant number of patients with type $1 \mathrm{GD}$ were switched to this drug following the shortage of imiglucerase [Giraldo et al. 2009]. A trial of miglustat in patients with type 3 GD on ERT did not conclusively show combination therapy (i.e. miglustat and ERT) to provide additional benefits [Schiffmann et al. 2008].

Side effects reported with the use of miglustat include gastrointestinal (GI) problems (mainly diarrhea in $80 \%$ of patients particularly during the initial exposure to treatment), tremor, and peripheral neuropathy [Hollak et al. 2009]. The diarrhea probably results from miglustat's inhibition of other glucosidases in the gut; these problems tend to diminish with ongoing therapy and respond well to a low-carbohydrate diet.

A second SRT formulation, eliglustat, has been shown to achieve therapeutic outcomes in treated type $1 \mathrm{GD}$ patients that are comparable to ERT [Lukina et al. 2010]. Barring any long-term safety consideration, eliglustat could potentially be considered as an initial therapeutic option, or maintenance therapy in GD patients after disease stability has been achieved with ERT. GI side effects were infrequently reported in study patients on eliglustat, although 4/26 patients had a nonsustained ventricular tachycardia [Lukina et al. 2010]. The relationship of the latter problem to study drug is being further examined in ongoing trials. Eliglustat is predominately metabolized by CYP450 2D6 (CYP2D6) and to lesser extent by CYP450 3A4 (CYP3A4); its full spectrum of potential drug interactions remains to be established. Unfortunately, eliglustat is a substrate of the drug transporter p-glycoprotein; as such, there is rapid drug efflux out of CSF into the systemic circulation, preventing sufficient drug buildup within the CNS. Thus, eliglustat is unlikely to alter course in patients with the neuronopathic forms of GD. However, modifications to the drug or its congeners may lead to better retention within the CSF and greater uptake by brain parenchyma and a corresponding positive result, but outcomes would need to be demonstrated in clinical trials.

For the MPS disorders, genistein, a component of soy extract, has been shown to reduce the accumulation of glycosaminoglycans (GAG) in human MPS I, II, IIIA and IIIB fibroblasts [Piotrowska et al. 2006]. Interestingly, these findings are achieved not by directly inhibiting GAG synthesis but through modulation of epidermal growth factor (EGF) which is involved with maximizing GAG synthesis. This approach is probably best described as substrate modulation therapy; a term also appropriate for approaches to modify GAGs into a form that allows its degradation by intact pathways to which it is diverted through the use of glycan inhibitors [Brown et al. 2007].

In an open-label study of 10 MPS patients given genistein for 12 months, there was a significant reduction in heparan sulfate levels in urine and improvements of hair morphology and in cognitive function [Jakóbkiewicz-Banecka et al. 2009]. No adverse reactions were observed. Additional studies are underway, to ascertain its safety and efficacy in patients with MPS III.

\section{Substrate depletion therapy}

In cystinosis, there is a defect of cysteine egress out of the lysosome, caused by mutations in the transmembrane transport protein cystinosin. The oral administration of an aminothiol cysteamine promotes the lowering of cystine content in lysosomes of patients with cystinosis. Cysteamine promotes an intralysosomal disulfide interchange and the formation of a conjugated form of 
cysteine which is able to leave the lysosome via other transporters. Treated patients show maintenance of renal function and improved growth [Kleta and Gahl, 2004]. In addition, cysteamine eyedrops help to dissolve the corneal crystals that can develop in these individuals, including postkidney transplant cases.

Chemical-chaperone enzyme

enhancement therapy

The use of pharmacological chaperones has been proposed as an alternative therapeutic approach for certain LSDs, based on a demonstration that particular agents acting as active site inhibitors can serve as a template to promote the refolding and correct trafficking of mutant proteins [Fan and Ishii, 2007]. As protein rescue may only occur in certain cases (e.g. missense mutations that do not lead to a major loss or destruction of the enzyme or inactivate the catalytic site), disease-specific treatments would be limited to a subset of patients and not the entire spectrum of enzyme deficient patients (such as those with null alleles or mutations leading to premature protein truncation).

Pharmacologic chaperones appear to work best in the presence of residual enzyme activity. As these are patients who may follow an atypical course of disease, it will be necessary to identify the subgroup of patients most likely to show disease progression and the appropriate time to introduce the treatment in these cases. This is a practical issue, not currently fully addressed by information regarding patient genotype or the monitoring of biomarkers. There is also uncertainty regarding the predictive value of in vitro screening tests. Furthermore, certain pharmacologic chaperones have inhibitory properties, which potentially complicate determination of the right combination of dose and frequency of drug administration to maximize enhancement of enzymes activity. There may also be differences in enzyme enhancement across cell types in individual patients that could result in variable clinical outcomes.

The safety and effectiveness of the use of pharmacologic chaperones remain to be established conclusively. Two formulations, isofagomine and migalastat, are the subject of clinical trials for GD and Fabry disease, respectively [Lieberman et al. 2009]. For both drugs, the rationale for advancing to human subjects was established through proof of principle studies in vitro using cells obtained from affected individuals and in animal models of disease. Obviously, it will be important to demonstrate that the use of pharmacologic chaperones not only leads to enhancement of enzyme activity, but also leads to a corresponding substantial decrease in substrate storage and ultimately clinical benefit.

Interestingly, studies in affected cells from Pompe disease patients suggest the use of pharmacologic chaperones may also improve uptake and stability of exogenous enzymes, thereby providing potential justification for therapy in combination with ERT [Porto et al. 2009].

Recently, it has been suggested that mutant proteins may be rescued from premature degradation through enhancement of other factors involved with promoting proper protein folding within the endoplasmic reticulum (ER). For instance, celastrol and MG 132 have been demonstrated to partially restore enzyme activity in cell lines obtained from patients with GD and TSD [Mu et al. 2008]. Also, verapamil and diltiazem, which act as calcium antagonists, partially restore lysosomal enzyme activity in cell lines from patients with GD, MPS IIIA (Sanfilippo syndrome) and mannosidosis [Ong et al. 2010]. Presumably, blocking calcium channels enhances the proper folding of mutant enzyme by upregulating a subset of molecular chaperones within the ER. The latter process has been referred to as proteostasis regulation, wherein protein rescue is mediated indirectly by upregulating endogenous chaperones rather than acting as a folding template [Mu et al. 2008]. Thus, in contrast to pharmacologic chaperones that are specific to particular mutant enzymes in any given disease, proteostasis regulators may serve a broad spectrum of disease: a highly desirable situation.

\section{Premature stop codon read through}

In several LSDs, the underlying defect is a premature stop codon mutation. Several drugs, such as gentamicin, have been shown to selectively target premature stop codons and modify the tRNA recognition process, essentially enabling the potential for read through and translation of the encoded protein [Brooks et al. 2006; Hein et al. 2004]. Obviously, putative agents will have to be carefully selected to ensure that processes involved with native stop codons of other genes are not dysregulated. 
In severe MPS I (Hurler syndrome) the premature stop codons Q70X and W402X are two of the most common $\alpha$-L-iduronidase (IDUA) gene mutations. In $\mathrm{CHO}-\mathrm{K} 1$ cells expressing these mutations, gentamicin enhanced stop codon read through and restored a low level of IDUA activity and reduced lysosomal GAG accumulation [Hein et al. 2004; Keeling et al. 2001].

There are currently no clinical trials to evaluate drugs that enable premature codon read through in patients with LSDs, although there remains great interest in exploring its potential role in these cases.

\section{Gene therapy}

Gene therapy remains a promising treatment approach for the LSDs. Although several animal studies suggest therapeutic benefits, application in human patients remains investigational. Several studies have been performed primarily in mouse models of disease, and in most cases with a viral-vector-mediated approach involving systemic and/or direct injection into CSF or brain parenchyma [Sands and Haskins, 2008]. Improvements have been observed in animal models of MPS IIIA, IIIB and VII, infantile neuronal ceroid lipofuscinosis (NCL), Niemann-Pick disease, metachromatic leukodystrophy, and Krabbe disease injected with either a lentiviral or an adeno-associated viral-vector and the relevant cognate gene [Di Domenico et al. 2009; Cearley and Wolfe 2007; Fraldi et al. 2007; Kurai et al. 2007; Passini et al. 2007; Rafi et al. 2005]. Meanwhile, there is growing interest in demonstrating proof of principle in larger animal models, such as sheep, cats and dogs [Haskins, 2009]. It is hoped these latter studies will help identify the hurdles that could be encountered in studies involving human subjects.

A trial to examine the safety of gene therapy in patients with late infantile NCL has been undertaken, using direct injection of AAV2 containing the CLN2 gene (encoding tripeptidyl-peptidase I) [Worgall et al. 2008]. Brain MRI showed a trend towards a lesser deterioration in atrophy and cortical apparent diffusion coefficient in contrast to the progression in the 18 months prior to the trial. The decline in the condition of the eight treated subjects for whom data were available for more than 6 months was shown to be significantly slower than that in a number of historical control groups.
Apart from the transduction of enzyme-deficient cells, modification of donor cells used in transplant may also enable overexpression of the cognate enzyme and enhance the benefit derived from HSCT alone. In studies involving the mouse model of metachromatic leukodystrophy, ex vivo gene transfer into hematopoietic cells led to improved outcomes when compared with transplant of unmodified cells [Biffi et al. 2004].

\section{Antisense splicing modulation therapy}

Certain mutations create novel splice sites, resulting in an altered transcript and dysfunctional protein product which is usually degraded prematurely. Antisense oligonucleotides (AOs) force the use of the natural splice sites, by modulating the splicing pattern through steric hindrance of the recognition and binding of the splicing apparatus to selected sequences [Du and Gatti, 2009]. As a consequence, there is greater recovery of normally spliced transcripts encoding the functional protein. Studies to date have been confined to experiments involving cells in culture and have not advanced to preclinical (animal model) trials.

In studies using cells from NPC patients, AO treatment has been shown to reverse aberrant splicing due to an intronic mutation (c.1554$1009 \mathrm{G}>$ A located in intron 9) which causes an inclusion of a pseudo-exon. The mutation in this case generates a premature termination codon and a transcript which is degraded by the nonsense-mediated mRNA decay mechanism. Normal splicing was restored in fibroblasts from the patient by a specific antisense morpholino oligonucleotide targeted to the cryptic splice site [Rodriguez-Pascau et al. 2009].

\section{Organ transplantation}

In LSDs associated with failure of the kidneys (e.g. Fabry disease, cystinosis), liver (GD) or heart (Fabry disease), organ transplantation has been undertaken as a life-shaving measure [Ayto et al. 2010; Karras et al. 2008; Nesterova and Gahl, 2008].

Although donor organs from living relatives or cadavers express normal enzyme activity and are spared from re-accumulation of substrate, it is unlikely that sufficient amounts of the enzyme are secreted or adequate microchimerism develops to effect clearance of the systemic disease burden. As with HSCT, it is important to exclude the possibility that the organ donor may be a 
carrier; in particular, one must avoid the transfer of a presumed healthy kidney from a female relative in a family segregating for Fabry disease (an X-linked trait).

In selected cases, where there is potential to improve quality of life and extend survival, organ transplantation may be undertaken. Obviously, one must take appropriate precautions for increased risks related to the systemic disease burden.

\section{Adjunctive therapies}

Increased understanding of downstream disease mechanisms implicated in LSD pathology may lead to identification of potential targets for therapeutic intervention [Bellettato and Scarpa, 2010; Vitner et al. 2010]. Animal studies suggest cellular changes develop prior to onset of disease manifestations and, thus, there may be a critical need for early diagnosis and intervention to optimize clinical outcome [Hemsley and Hopwood, 2010]. However, pathology may be initiated prenatally, in which case even newborn screening to identify affected individuals may not be adequate. Obviously, in the latter instance identification of families at risk may enable preimplantation genetic or prenatal diagnosis to reduce recurrence risk. There is also potential for treatment in utero that may alter prognosis, although this remains to be demonstrated.

Aberrant inflammation has been described in several LSDs including GM1-gangliosidosis, GM2 gangliosidosis (Sandhoff disease) and NPC [Jeyakumar et al. 2003]. In mouse models of Sandhoff disease and NPC, nonsteroidal antiinflammatory agents reduced brain inflammation and improved motor function and survival when given alone or in combination with miglustat [Jeyakumar et al. 2004, 1999]. Also, in the mouse model for NPC the use of the c-Ablspecific inhibitor imatinib resulted in improved neurological symptoms [Alvarez et al. 2008].

Osteopenia has been described in GD, Fabry disease and Pompe disease [van den Berg et al. 2010; Germain et al. 2005; Pastores et al. 1996]. Anti-bone-resorbing agents, such as alendronate, have been shown to improve bone density in GD patients receiving ERT [Wenstrup et al. 2004]. Such drugs may also be useful in reducing fracture risks in Fabry and Pompe disease patients with severe osteopenia.
In Fabry disease, the use of angiotensin-converting enzyme inhibitors has been advocated in patients with proteinuria [Ortiz et al. 2008; Tahir et al. 2007]. In addition, antiplatelet aggregating agents have been recommended to reduce risk of developing stroke.

Thus, adjunctive therapies may be useful in combination with available targeted treatments for improved results, although it would be ideal for controlled studies to be undertaken prior to general implementation. Longitudinal follow up with data collected through registry programs and outcomes compared with a historical data set may be acceptable, given the understandable difficulty of conducting trials in rare diseases and ethical concerns about withholding treatments that may be lead to added benefits.

\section{Disease prevention}

We should not overlook strategies aimed at disease prevention through carrier detection and appropriate genetic counseling. In addition to newborn screening programs to prevent recurrence, families at risk have the option of pre-implantation genetic diagnosis and prenatal diagnosis [Altarescu et al. 2010; Hwu et al. 2010]. The effectiveness of these programs has been demonstrated for TSD in the Ashkenazi Jewish community wherein the incidence of the disorder has declined significantly [Kaback, 2001].

\section{Conclusion}

The field of LSDs is being rapidly transformed by advances in our understanding of disease mechanisms and the introduction of various therapeutic options. None of these approaches are necessarily exclusive, but hold the promise of introducing combination therapies to improve clinical outcome. However, it will be critical to initially demonstrate that singular strategies do alter disease course. Obviously, clinical trials will have to demonstrate that there is indeed added benefit to be gained with a combination approach. The latter is not a trivial issue, as current and projected costs of care for patients with LSDs are huge, and the conduct of trials can be very expensive, given the rarity of these diseases. Approaches, such as SRT and proteostatis regulation, which may be potentially useful in a broad spectrum of disorders, spreads the cost of development, and may help to address concerns raised by third-party payers. Meanwhile, significant in roads have not been made in the management of 
primary CNS disease; primarily through supportive care which can improve the lives of affected individuals and their families. One can be hopeful that therapeutic strategies that can have an impact on ultimate neurologic course will eventually be developed.

\section{Funding}

This article received no specific grant from any funding agency in the public, commercial, or notfor-profit sectors.

\section{Conflict of interest statement}

GM Pastores is the recipient of research grants from Actelion, Amicus, Biomarin, Genzyme, Protalix and Shire HGT; pharmaceutical/biotechnology companies engaged in drug development programs for lysosomal storage disorders.

\section{References}

Altarescu, G., Renbaum, P., Eldar-Geva, T., Varshower, I., Brooks, B., Beeri, R. et al. (2010) Preimplantation genetic diagnosis (PGD) for a treatable disorder: Gaucher disease type 1 as a model. Blood Cells Mol Dis in press.

Alvarez, A.R., Klein, A., Castro, J., Cancino, G.I., Amigo, J., Mosqueira, M. et al. (2008) Imatinib therapy blocks cerebellar apoptosis: improves neurological symptoms in a mouse model of Niemann-Pick type C disease. FASEB $\mathcal{F}$ 22: 3617-3627.

Araya, K., Sakai, N., Mohri, I., Kagitani-Shimono, K., Okinaga, T., Hashii, Y. et al. (2009) Localized donor cells in brain of a Hunter disease patient after cord blood stem cell transplantation. Mol Genet Metab 98: 255-263.

Ayto, R.M., Hughes, D.A., Jeevaratnam, P., Rolles, K., Burroughs, A.K., Mistry, P.K. et al. (2010) Long-term outcomes of liver transplantation in type 1 Gaucher disease. Am f Transplant 10: 1934-1939.

Bellettato, C.M. and Scarpa, M. (2010) Pathophysiology of neuropathic lysosomal storage disorders. F Inherit Metab Dis 33: 347-362.

Biffi, A., De Palma, M., Quattrini, A., Del Carro, U., Amadio, S., Visigalli, I. et al. (2004) Correction of metachromatic leukodystrophy in the mouse model by transplantation of genetically modified hematopoietic stem cells. f Clin Invest 113: 1118-1129.

Brooks, D.A., Muller, V.J. and Hopwood, J.J. (2006) Stop-codon read-through for patients affected by a lysosomal storage disorder. Trends Mol Med 12: 367-373.

Brown, J.R., Crawford, B.E. and Esko, J.D. (2007) Glycan antagonists and inhibitors: a fount for drug discovery. Crit Rev Biochem Mol Biol 42: 481-515.
Caballero, L., Climent, V., Hernández-Romero, D., Quintanilla, M.A., de la Morena, G. and Marín, F. (2010) Enzyme replacement therapy in Fabry disease: influence on cardiac manifestations. Curr Med Chem 17: 1679-1689.

Cearley, C.N. and Wolfe, J.H. (2007) A single injection of an adeno-associated virus vector into nuclei with divergent connections results in widespread vector distribution in the brain and global correction of a neurogenetic disease. $\mathcal{F}$ Neurosci 27: 9928-9940.

Cox, T., Lachmann, R., Hollak, C., Aerts, J., van Weely, S., Hrebícek, M. et al. (2000) Novel oral treatment of Gaucher's disease with N-butyldeoxynojirimycin (OGT 918): to decrease substrate biosynthesis. Lancet 355: 1481-1485.

Di Domenico, C., Villani, G.R., Di Napoli, D., Nusco, E., Cali, G., Nitsch, L. et al. (2009) Intracranial gene delivery of LV-NAGLU vector corrects neuropathology in murine MPS IIIB. Am $\mathcal{F}$ Med Genet 149A: 1209-1218.

Dickson, P., McEntee, M., Vogler, C., Le, S., Levy, B., Peinovich, M. et al. (2007) Intrathecal enzyme replacement therapy: successful treatment of brain disease via the cerebrospinal fluid. Mol Genet Metab 91: 61-68.

Du, L. and Gatti, R.A. (2009) Progress toward therapy with antisense-mediated splicing modulation. Curr Opin Mol Ther 11: 116-123.

Fan, J.Q. and Ishii, S. (2007) Active-site-specific chaperone therapy for Fabry disease. Yin and Yang of enzyme inhibitors. FEBS $\mathcal{F}$ 274: 4962-4971.

Fraldi, A., Hemsley, K., Crawley, A., Lombardi, A., Lau, A., Sutherland, L. et al. (2007) Functional correction of CNS lesions in an MPS-IIIA mouse model by intracerebral AAV-mediated delivery of sulfamidase and SUMF1 genes. Hum Mol Genet 16: 2693-2702.

Fratantoni, J.C., Hall, C.W. and Neufeld, E.F. (1968) Hurler and Hunter syndromes: mutual correction of the defect in cultured fibroblasts. Science 162: 570-572.

Germain, D.P., Benistan, K., Boutouyrie, P. and Mutschler, C. (2005) Osteopenia and osteoporosis: previously unrecognized manifestations of Fabry disease. Clin Genet 68: 93-95.

Giraldo, P., Alfonso, P., Atutxa, K., Fernández-Galán, M.A., Barez, A., Franco, R. et al. (2009) Real-world clinical experience with long-term miglustat maintenance therapy in type 1 Gaucher disease: the ZAGAL project. Haematologica 94: 1771-1775.

Grubb, J.H., Vogler, C., Levy, B., Galvin, N., Tan, Y. and Sly, W.S. (2008) Chemically modified beta-glucuronidase crosses blood-brain barrier and clears neuronal storage in murine mucopolysaccharidosis VII. Proc Natl Acad Sci U S A 105: 2616-2621.

Haskins, M. (2007) Animal models for mucopolysaccharidosis disorders and their clinical relevance. Acta Paediatr Suppl 96: 56-62. 
Haskins, M. (2009) Gene therapy for lysosomal storage diseases (LSDs) in large animal models. ILAR $\mathcal{F}$ 50: $112-121$.

Hein, L.K., Bawden, M., Muller, V.J., Sillence, D., Hopwood, J.J. and Brooks, D.A. (2004) alphaLiduronidase premature stop codons and potential read-through in mucopolysaccharidosis type I patients. f Mol Biol 338: 453-462.

Hemsley, K.M. and Hopwood, J.J. (2010) Lessons learnt from animal models: pathophysiology of neuropathic lysosomal storage disorders. F Inherit Metab Dis 33: $363-371$.

Hemsley, K.M., Luck, A.J., Crawley, A.C., Hassiotis, S., Beard, H., King, B. et al. (2009) Examination of intravenous and intra-CSF protein delivery for treatment of neurological disease. Eur $\mathcal{F}$ NeuroSci 29: 1197-1214.

Hollak, C.E., Hughes, D., van Schaik, I.N., Schwierin, B. and Bembi, B. (2009) Miglustat (Zavesca) in type 1 Gaucher disease: 5-year results of a post-authorisation safety surveillance programme. Pharmacoepidemiol Drug Saf 18: 770-777.

Hopwood, J. and Ebner, R. (2009) Disease pathogenesis-clinical interpretation. Int 7 Clin Pharmacol Ther 47(Suppl 1): S41.

Hwu, W.L., Chien, Y.H. and Lee, N.C. (2010)

Newborn screening for neuropathic lysosomal storage disorders. F Inherit Metab Dis 33: 381-386.

Jakóbkiewicz-Banecka, J., Piotrowska, E., Narajczyk, M., Barańska, S. and Wegrzyn, G. (2009) Genisteinmediated inhibition of glycosaminoglycan synthesis, which corrects storage in cells of patients suffering from mucopolysaccharidoses, acts by influencing an epidermal growth factor-dependent pathway. $\mathcal{F}$ Biomed Sci 16: 26

Jardim, L.B., Villanueva, M.M., de Souza, C.F. and Netto, C.B. (2010) Clinical aspects of neuropathic lysosomal storage disorders. F Inherit Metab Dis 33: 315-329.

Jeyakumar, M., Butters, T.D., Cortina-Borja, M., Hunnam, V., Proia, R.L., Perry, V.H. et al. (1999) Delayed symptom onset and increased life expectancy in Sandhoff disease mice treated with N-butyldeoxynojirimycin. Proc Natl Acad Sci U S A 96: 6388-6393.

Jeyakumar, M., Smith, D.A., Williams, I.M., Borja, M.C., Neville, D.C., Butters, T.D. et al. (2004) NSAIDs increase survival in the Sandhoff disease mouse: synergy with Nbutyldeoxynojirimycin. Ann Neurol 56: 642-649.

Jeyakumar, M., Thomas, R., Elliot-Smith, E., Smith, D.A., van der Spoel, A.C., d'Azzo, A. et al. (2003) Central nervous system inflammation is a hallmark of pathogenesis in mouse models of GM1 and GM2 gangliosidosis. Brain 126: 974-987.

Kaback, M.M. (2001) Screening and prevention in Tay-Sachs disease: origins, update, and impact. $A d v$ Genet 44: 253-265.
Karras, A., De Lentdecker, P., Delahousse, M., Debauchez, M., Tricot, L., Pastural, M. et al. (2008) Combined heart and kidney transplantation in a patient with Fabry disease in the enzyme replacement therapy era. Am $\mathcal{F}$ Transplant 8: 1345-1348.

Keeling, K.M., Brooks, D.A., Hopwood, J.J., Li, P., Thompson, J.N. and Bedwell, D.M. (2001)

Gentamicin-mediated suppression of Hurler syndrome stop mutations restores a low level of alphaL-iduronidase activity and reduces lysosomal glycosaminoglycan accumulation. Hum Mol Genet 10: $291-299$.

Kishnani, P.S., Goldenberg, P.C., DeArmey, S.L., Heller, J., Benjamin, D., Young, S. et al. (2010) Crossreactive immunologic material status affects treatment outcomes in Pompe disease infants. Mol Genet Metab 99: 26-33.

Krivit, W., Sung, J.H., Shapiro, E.G. and Lockman, L.A. (1995) Microglia: the effector cell for reconstitution of the central nervous system following bone marrow transplantation for lysosomal and peroxisomal storage diseases. Cell Transplant 4: 385-392.

Kleta, R. and Gahl, W.A. (2004) Pharmacological treatment of nephropathic cystinosis with cysteamine. Expert Opin Pharmacother 5: 2255-2262.

Kurai, T., Hisayasu, S., Kitagawa, R., Migita, M., Suzuki, H., Hirai, Y. et al. (2007) AAV1 mediated co-expression of formylglycine-generating enzyme and arylsulfatase A efficiently corrects sulfatide storage in a mouse model of metachromatic leukodystrophy. Mol Ther 15: 38-43.

Lau, A.A., Hannouche, H., Rozaklis, T., Hassiotis, S. Hopwood, J.J. and Hemsley, K.M. (2010) Allogeneic stem cell transplantation does not improve neurological deficits in mucopolysaccharidosis type IIIA mice. Exp Neurol in press.

Lieberman, R.L., D'aquino, J.A., Ringe, D. and Petsko, G.A. (2009) Effects of $\mathrm{pH}$ and iminosugar pharmacological chaperones on lysosomal glycosidase structure and stability. Biochemistry 48: 4816-4827.

Linthorst, G.E., Hollak, C.E., Donker-Koopman, W.E., Strijland, A. and Aerts, J.M. (2004) Enzyme therapy for Fabry disease: neutralizing antibodies toward agalsidase alpha and beta. Kidney Int 66: 1589-1595.

Lukina, E., Watman, N., Arreguin, E.A., Banikazemi, M., Dragosky, M., Iastrebner, M., et al. (2010) A phase 2 study of eliglustat tartrate (Genz-112638), an oral substrate reduction therapy for Gaucher disease type 1 . Blood in press.

Maegawa, G.H., Banwell, B.L., Blaser, S., Sorge, G., Toplak, M., Ackerley, C. et al. (2009) Substrate reduction therapy in juvenile GM2 gangliosidosis. Mol Genet Metab 98: 215-224.

Malm, G., Gustafsson, B., Berglund, G., Lindstrom, M., Naess, K., Borgstrom, B. et al. (2008) Outcome in six children with mucopolysaccharidosis type IH, Hurler syndrome, after haematopoietic stem cell 
transplantation (HSCT). Acta Paediatr 97: 1108-1112.

Mehta, A., Beck, M., Elliott, P., Giugliani, R., Linhart, A., Sunder-Plassmann, G. et al. (2009) Enzyme replacement therapy with agalsidase alfa in patients with Fabry's disease: an analysis of registry data. Lancet 374(9706): 1986-1996.

Miyake, N., Miyake, K., Karlsson, S. and Shimada, T. (2010) Successful treatment of metachromatic leukodystrophy using bone marrow transplantation of HoxB4 overexpressing cells. Mol Ther 18: 1373-1378.

Mu, T.W., Ong, D.S., Wang, Y.J., Balch, W.E., Yates III, J.R., Segatori, L. et al. (2008) Chemical and biological approaches synergize to ameliorate proteinfolding diseases. Cell 134: 769-781.

Munoz-Rojas, M.V., Vieira, T., Costa, R., Fagondes, S., John, A., Jardim, L.B. et al. (2008) Intrathecal enzyme replacement therapy in a patient with mucopolysaccharidosis type I and symptomatic spinal cord compression. Am F Med Genet 146A: 2538-2544.

Nesterova, G. and Gahl, W. (2008) Nephropathic cystinosis: late complications of a multisystemic disease. Pediatr Nephrol 23: 863-878.

Ong, D.S., Mu, T.W., Palmer, A.E. and Kelly, J.W. (2010) Endoplasmic reticulum $\mathrm{Ca} 2+$ increases enhance mutant glucocerebrosidase proteostasis. Nat Chem Biol 6: 424-432.

Orchard, P.J., Blazar, B.R., Wagner, J., Charnas, L., Krivit, W. and Tolar, J. (2007) Hematopoietic cell therapy for metabolic disease. F Pediatr 151: 340-346.

Ortiz, A., Oliveira, J.P., Wanner, C., Brenner, B.M., Waldek, S. and Warnock, D.G. (2008)

Recommendations and guidelines for the diagnosis and treatment of Fabry nephropathy in adults. Nat Clin Pract Nephrol 4: 327-336.

Parkinson-Lawrence, E.J., Shandala, T., Prodoehl, M., Plew, R., Borlace, G.N. and Brooks, D.A. (2010) Lysosomal storage disease: revealing lysosomal function and physiology. Physiology (Bethesda) 25: $102-115$.

Passini, M.A., Bu, J., Fidler, J.A., Ziegler, R.J., Foley, J.W., Dodge, J.C. et al. (2007) Combination brain and systemic injections of AAV provide maximal functional and survival benefits in the Niemann-Pick mouse. Proc Natl Acad Sci U S A 104: 9505-9510.

Pastores, G.M., Barnett, N.L. and Kolodny, E.H. (2005) An open-label, noncomparative study of miglustat in type I Gaucher disease: efficacy and tolerability over 24 months of treatment. Clin Ther 27: 1215-1227.

Pastores, G.M., Wallenstein, S., Desnick, R.J. and Luckey, M.M. (1996) Bone density in Type 1 Gaucher disease. F Bone Miner Res 11: 1801-1807.

Patterson, M.C., Vecchio, D., Prady, H., Abel, L. and Wraith, J.E. (2007) Miglustat for treatment of Niemann-Pick C disease: a randomised controlled study. Lancet Neurol 6: 765-772.
Piotrowska, E., Jakóbkiewicz-Banecka, J., Barańska, S., Tylki-Szymańska, A., Czartoryska, B., Wegrzyn, A. et al. (2006) Genistein-mediated inhibition of glycosaminoglycans synthesis as a basis for gene expressiontargeted isoflavone therapy for mucopolysaccharidoses. Eur F Hum Genet 14: 846-852.

Platt, F.M. and Jeyakumar, M. (2008) Substrate reduction therapy. Acta Paediatr Suppl 97: 88-93.

Porto, C., Cardone, M., Fontana, F., Rossi, B., Tuzzi, M.R., Tarallo, A. et al. (2009) The pharmacological chaperone N-butyldeoxynojirimycin enhances enzyme replacement therapy in Pompe disease fibroblasts. $\mathrm{Mol}$ Ther 17: 964-971.

Prasad, V.K. and Kurtzberg, J. (2008)

Emerging trends in transplantation of inherited metabolic diseases. Bone Marrow Transplant 41(2): 99-108.

Prasad, V.K. and Kurtzberg, J. (2010a) Transplant outcomes in mucopolysaccharidoses. Semin Hematol 47: 59-69.

Prasad, V.K. and Kurtzberg, J. (2010b) Cord blood and bone marrow transplantation in inherited metabolic diseases: scientific basis, current status and future directions. Br F Haematol 148(3): 356-372.

Rafi, M.A., Zhi Rao, H., Passini, M.A., Curtis, M., Vanier, M.T., Zaka, M. et al. (2005) AAVmediated expression of galactocerebrosidase in brain results in attenuated symptoms and extended life span in murine models of globoid cell leukodystrophy. Mol Ther 11: 734-744.

Rodriguez-Pascau, L., Coll, M.J., Vilageliu, L. and Grinberg, D. (2009) Antisense oligonucleotide treatment for a pseudoexon generating mutation in the NPC1 gene causing Niemann-Pick type C disease. Hum Mutat 30: E993-E1001.

Sands, M.S. and Haskins, M.E. (2008) CNS-directed gene therapy for lysosomal storage diseases. Acta Paediatr Suppl 97: 22-27.

Savas, P.S., Hemsley, K.M. and Hopwood, J.J. (2004) Intracerebral injection of sulfamidase delays neuropathology in murine MPS-IIIA. Mol Genet Metab 82: $273-285$.

Schiffmann, R., Fitzgibbon, E.J., Harris, C., DeVile, C., Davies, E.H., Abel, L. et al. (2008) Randomized, controlled trial of miglustat in Gaucher's disease type 3. Ann Neurol 64: 514-522.

Schiffmann, R., Heyes, M.P., Aerts, J.M., Dambrosia, J.M., Patterson, M.C., DeGraba, T. et al. (1997) Prospective study of neurological responses to treatment with macrophage-targeted glucocerebrosidase in patients with type 3 Gaucher's disease. Ann Neurol 42: 613-621.

Shapiro, B.E., Pastores, G.M., Gianutsos, J., Luzy, C. and Kolodny, E.H. (2009) Miglustat in late-onset Tay-Sachs disease: a 12-month, randomized, controlled clinical study with 24 months of extended treatment. Genet Med 11: 425-433. 
Spencer, B.J. and Verma, I.M. (2007) Targeted delivery of proteins across the blood-brain barrier. Proc Natl Acad Sci U S A 104: 7594-7599.

Starzyk, K., Richards, S., Yee, J., Smith, S.E. and Kingma, W. (2007) The long-term international safety experience of imiglucerase therapy for Gaucher disease. Mol Genet Metab 90: 157-163.

Sun, B., Bird, A., Young, S.P., Kishnani, P.S., Chen, Y.T. and Koeberl, D.D. (2007) Enhanced response to enzyme replacement therapy in Pompe disease after the induction of immune tolerance. Am $\mathcal{F}$ Hum Genet 81: 1042-1049.

Tahir, H., Jackson, L.L. and Warnock, D.G. (2007) Antiproteinuric therapy and Fabry nephropathy: sustained reduction of proteinuria in patients receiving enzyme replacement therapy with agalsidase-beta. f Am Soc Nephrol 18: 2609-2617.

Tallaksen, C.M. and Berg, J.E. (2009) Miglustat therapy in juvenile Sandhoff disease. $\mathcal{F}$ Inherit Metab Dis in press.

Tolar, J., Grewal, S.S., Bjoraker, K.J., Whitley, C.B., Shapiro, E.G., Charnas, L. et al. (2008) Combination of enzyme replacement and hematopoietic stem cell transplantation as therapy for Hurler syndrome. Bone Marrow Transplant 41: 531-535.

van den Berg, L.E., Zandbergen, A.A., van Capelle, C.I., de Vries, J.M., Hop, W.C., van den Hout, J.M. et al. (2010) Low bone mass in Pompe disease Muscular strength as a predictor of bone mineral density. Bone in press.

Visit SAGE journals online http://tae.sagepub.com

(SSAGEJOURNALS Online van der Ploeg, A.T., Clemens, P.R., Corzo, D., Escolar, D.M., Florence, J., Groeneveld, G.J. et al. (2010) A randomized study of alglucosidase alfa in late-onset Pompe's disease. $N$ Engl f Med 362(15): 1396-1406.

Vitner, E.B., Platt, F.M. and Futerman, A.H. (2010) Common and uncommon pathogenic cascades in lysosomal storage diseases. $7 \mathrm{Biol}$ Chem 285: 20423-20427.

Wada, R., Tifft, C.J. and Proia, R.L. (2000) Microglial activation precedes acute neurodegeneration in Sandhoff disease and is suppressed by bone marrow transplantation. Proc Natl Acad Sci U S A 97: 10954-10959.

Wang, D., Zhang, W., Kalfa, T.A., Grabowski, G., Davies, S., Malik, P. et al. (2009) Reprogramming erythroid cells for lysosomal enzyme production leads to visceral and CNS cross-correction in mice with Hurler syndrome. Proc Natl Acad Sci U S A 106: 19958-19963.

Wenstrup, R.J., Bailey, L., Grabowski, G.A., Moskovitz, J., Oestreich, A.E., Wu, W. et al. (2004) Gaucher disease: alendronate disodium improves bone mineral density in adults receiving enzyme therapy. Blood 104: 1253-1257.

Worgall, S., Sondhi, D., Hackett, N.R., Kosofsky, B., Kekatpure, M.V., Neyzi, N. et al. (2008) Treatment of late infantile neuronal ceroid lipofuscinosis by CNS administration of a serotype 2 adeno-associated virus expressing CLN2 cDNA. Hum Gene Ther 19: 463-474.

Wortmann, S.B., Lefeber, D.J., Dekomien, G., Willemsen, M.A., Wevers, R.A. and Morava, E. (2009) Substrate deprivation therapy in juvenile Sandhoff disease. 7 Inherit Metab Dis in press. 